\title{
Domain state model for exchange bias. II. Experiments
}

\author{
J. Keller, P. Miltényi, B. Beschoten,* and G. Güntherodt \\ 2.Physikalisches Institut, RWTH Aachen, 52056 Aachen, Germany \\ U. Nowak ${ }^{\dagger}$ and K. D. Usadel \\ Theoretische Tieftemperaturphysik, Gerhard-Mercator-Universität Duisburg, 47048 Duisburg, Germany
}

(Received 15 August 2001; published 17 July 2002)

\begin{abstract}
The exchange bias coupling at ferro-/antiferromagnetic interfaces of epitaxially grown $\mathrm{Co} / \mathrm{CoO}$ bilayers can be intentionally enhanced and controlled by diluting the antiferromagnetic $\mathrm{CoO}$ layer, i.e., by introducing (i) nonmagnetic substitutions $\left(\mathrm{Co}_{1-x} \mathrm{Mg}_{x} \mathrm{O}\right)$ or (ii) Co deficiencie $\left(\mathrm{Co}_{1-y} \mathrm{O}\right)$. All intentional nonmagnetic cations or defects were placed away from the interface throughout the whole volume part of the antiferromagnetic layer. This way the roughness at the $\mathrm{Co} / \mathrm{CoO}$ interface was kept practically the same. For both types of defects, the exchange bias fiel can be increased by a factor of 3 to 4 . Hence, exchange bias is primarily not due to roughness at the interface but rather can be controlled by the defects in the volume part of the antiferromagnetic layer. We systematically investigate the dilution dependence of various phenomena of exchange bias, such as the vertical magnetization shift of the hysteresis loop, temperature dependence, training effect, cooling fiel dependence, and antiferromagnetic layer thickness dependence. All these phenomena are directly compared to results from Monte Carlo simulations and are shown to be consistently described by the domain state model for exchange bias. The combined experimental and theoretical finding suggest that the origin of exchange bias in $\mathrm{Co} / \mathrm{CoO}$ results from a domain state in the volume part of the antiferromagnet stabilized by the defects.
\end{abstract}

PACS number(s): 75.70.Cn, 75.70.Kw, 75.30.Gw, 75.50.Lk

\section{INTRODUCTION}

Direct exchange coupling at the interface between a ferromagnetic (FM) and an antiferromagnetic (AFM) layer may result in exchange biasing, which induces an unidirectional anisotropy of the FM layer. The unidirectional anisotropy causes a shift of the hysteresis loop along the magnetic fiel axis. The magnitude of the fiel shift is called the exchange bias (EB) fiel $B_{\mathrm{EB}}$. Usually, the EB shift occurs after cooling the system with a saturated FM layer below the Néel temperature of the AFM layer or by layer deposition in an external magnetic field Despite four decades of research since its discovery ${ }^{1,2}$ and the commercially available magnetic sensor devices, ${ }^{3,4}$ the microscopic understanding of the EB effect is still not fully established. ${ }^{5}$

In a recent paper, ${ }^{6}$ we reported on $\mathrm{EB}$ studies in $\mathrm{Co} / \mathrm{CoO}$ bilayers as a function of volume defects in the antiferromagnet. Of particular importance in this study was the observation that nonmagnetic defects in the volume of the AFM layer can enhance the exchange bias by a factor of up to 3 . The nonmagnetic defects (dilution) in $\mathrm{CoO}$ were realized in two ways: (i) by overoxidizing $\mathrm{CoO}$ leading to $\mathrm{Co}$ deficien cies in $\mathrm{Co}_{1-y} \mathrm{O}$ or (ii) by substituting nonmagnetic $\mathrm{Mg}$ ions for magnetic $\mathrm{Co}$ in $\mathrm{Co}_{1-x} \mathrm{Mg}_{x} \mathrm{O}$. For all samples investigated a 0.4-nm-thick $\mathrm{CoO}$ layer with minimum defect concentration was placed at the interface. Therefore, in these systems the observed EB is primarily not due to disorder or defects at the interface. Rather, the strong dependence of the EB fiel on the dilution of the AFM layer was concluded to have its origin in the formation of a domain state in the volume of the AFM layer. This domain state gives rise to a small but significan excess of magnetic moments at the FM/AFM interface, which is irreversible under FM magnetization reversal.
These uncompensated moments cause and control exchange bias.

The "domain state" (DS) model gives a description of exchange bias, which is supported by Monte Carlo (MC) simulations. $^{6,7}$ The model links the physics of diluted antiferromagnets in an external fiel (Refs. 8 and 9) to the coupling mechanisms of exchange-coupled magnetic layers.

Malozemoff has already pointed out the relevance of AFM domains for the exchange bias effect due to interface roughness. ${ }^{10-12}$ In the DS model, however, the stabilization of AFM domains is not due to interfacial roughness, but rather induced and stabilized by the existence of volume defects in the AFM layer. Indeed, it appears reasonable to assume that any deviation from a perfect AFM crystalline structure (disorder in the AFM layer), such as defects, or grain or twin boundaries, can favor the formation of magnetic domains and by that affect the EB coupling. Here, nonmagnetic volume defects (dilution) in the antiferromagnet were chosen since the degree of dilution can easily and reproducibly be adjusted during sample deposition by the AFM stoichiometry.

The magnetic linear dichroism effect in soft-x-ray absorption has recently been used to probe as well as to image the domains and the structure in AFM films ${ }^{13-16}$ This spectroscopy technique has also been employed to image both FM and AFM domains in FM/AFM exchange-coupled systems. ${ }^{17-20}$ These domains have been shown to be coinciding and give evidence of EB coupling on a local scale.

In the present paper we systematically investigate a variety of the most characteristic EB phenomena for both $\mathrm{Co} / \mathrm{Co}_{1-x} \mathrm{Mg}_{x} \mathrm{O}$ and $\mathrm{Co} / \mathrm{Co}_{1-y} \mathrm{O}$ bilayer systems as a function of dilution. These phenomena include, besides EB fiel and coercivity field the shape and vertical shift of the hys- 
teresis loops, the temperature dependence of the EB field the training effect, the cooling fiel dependence, and the dependence of the EB on the AFM layer thickness. Each of these phenomena as a function of dilution is directly compared with results from MC simulations and discussed within the DS model, which is shown to provide a consistent description of the origin of EB as well as of a variety of related phenomena.

The paper is organized as follows. The following section describes the sample preparation by molecular-beam epitaxy (MBE). In Sec. III the dilution dependence of the structural properties is investigated by reflectio high-energy electron diffraction (RHEED), high-angle x-ray diffraction, and atomic force microscopy. Typical EB phenomena are presented in Sec. IV. For easier comparison, these effects are discussed in exactly the same order as in the preceding theoretical part of the paper. ${ }^{7}$ Finally, we conclude in the last section.

\section{SAMPLE PREPARATION}

The samples were prepared on (0001)-oriented sapphire substrates in a MBE chamber. Prior to fil deposition the substrates were heated to $T=775 \mathrm{~K}$ for $1 \mathrm{~h}$ in order to outgas the substrate holder and then cooled to the Co growth temperature of $T_{\mathrm{Co}}=575 \mathrm{~K}$. The layered sample structure is schematically illustrated in the inset of Fig. 3(a) below. First, a 6-nm-thick Co layer was deposited by electron-beam evaporation at a rate of $0.2 \mathrm{~nm} / \mathrm{min}$, which was subsequently annealed at a temperature of $T=775 \mathrm{~K}$ for $10 \mathrm{~min}$. Thereafter, a 0.4-nm-thick $\mathrm{CoO}$ layer was deposited for all samples at a substrate temperature of $T_{\text {oxide }}=350 \mathrm{~K}$ and an oxygen pressure of $p\left(\mathrm{O}_{2}\right)=3.3 \times 10^{-7} \mathrm{mbar}$ with a rate of $0.3 \mathrm{~nm} /$ min. Note that the RHEED pattern (see Sec. III) of the Co layer completely disappears after initially exposing the Co layer to an oxygen pressure of $p\left(\mathrm{O}_{2}\right)=3.3 \times 10^{-7} \mathrm{mbar}$ prior to $\mathrm{CoO}$ deposition. This demonstrates that the $\mathrm{Co} / \mathrm{CoO}$ interface is created over the entire Co layer even before depositing the $0.4 \mathrm{~nm} \mathrm{CoO}$ interface layer, which then ensures a continuous and homogeneous $\mathrm{CoO}$ layer at the FM/AFM interface. This procedure leads to practically identical FM/ AFM interfaces for all samples investigated at the lowest possible interface defect concentration, independent of the dilution of the following 20-nm-thick AFM layer.

On top of this $\mathrm{CoO}$ interface layer two different sets of diluted antiferromagnetic layers were deposited. In a firs set of samples $\mathrm{CoO}$ was diluted with nonmagnetic $\mathrm{MgO}$ forming $\mathrm{Co}_{1-x} \mathrm{Mg}_{x} \mathrm{O}$. Co and $\mathrm{MgO}$ were coevaporated in an oxygen atmosphere of $p\left(\mathrm{O}_{2}\right)=3.3 \times 10^{-7}$ mbar at a substrate temperature of $T_{\text {oxide }}=350 \mathrm{~K}$ and a deposition rate of $0.3 \mathrm{~nm} /$ min. The Mg concentration was varied between $x=0$ and $x$ $=1$, while the AFM layer thickness was kept constant at 20 $\mathrm{nm}$. For a second set of samples, nonmagnetic defects were realized by overoxidation of $\mathrm{CoO}$ yielding $\mathrm{Co}$-deficien $\mathrm{Co}_{1-y} \mathrm{O}$. The Co deficienc $y$ was controlled by varying the oxygen pressure during evaporation between $p\left(\mathrm{O}_{2}\right)=3.3$ $\times 10^{-7}$ mbar and $1.0 \times 10^{-5}$ mbar, while growth temperature, growth rate, and AFM layer thickness were all identical to the firs set of samples.
Two additional sets of samples were prepared to study the dependence of EB on the AFM layer thickness. For these samples the AFM interface layer was similarly prepared as described above, although this time we did not deposit the initial 0.4-nm-thick $\mathrm{CoO}$ layer. The AFM layer thickness was varied between $1 \mathrm{~nm}$ and $50 \mathrm{~nm}$. The two sets of samples were prepared with low $\left[p\left(\mathrm{O}_{2}\right)=3.3 \times 10^{-7} \mathrm{mbar}\right]$ and with intermediate $\left[p\left(\mathrm{O}_{2}\right)=5 \times 10^{-6} \mathrm{mbar}\right]$ oxygen pressure during evaporation. All thicknesses and the roughness of the different layers were controlled by a calibrated quartz microbalance and ex situ atomic force microscopy, respectively.

\section{STRUCTURAL PROPERTIES}

All samples were characterized in situ by RHEED and low energy electron diffraction (LEED). The RHEED patterns of the substrate and of the different layers of which the samples consist are depicted in Figs. 1(a)-1(d). The left panels show the diffraction patterns for the electron beam inci-

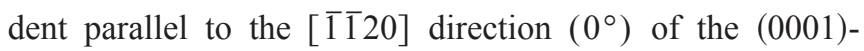
oriented sapphire substrate and the right panels for the beam parallel to the [1010] direction $\left(30^{\circ}\right)$. The spot separation in the RHEED image of the sapphire substrate in the $30^{\circ}$ direction [Fig. 1(a), right panel] is about $\sqrt{3}$ times the spot separation in the $0^{\circ}$ direction [Fig. 1(a), left panel]. This is consistent with the hexagonal surface symmetry of the sapphire (0001) substrate. ${ }^{21}$ For a 6-nm-thick Co layer on the sapphire substrate in Fig. 1(b) the same ratio is found but the two directions are interchanged. From this we conclude that Co grows epitaxially in either fcc (111) or in hcp (0001) orientation, which only differ in their so-called $\mathrm{ABAB}$ or $\mathrm{ABCABC}$ stacking order along the surface normal. ${ }^{21}$ Additional LEED investigations (not shown) reveal clearly the corresponding sixfold symmetry.

RHEED images of the 20-nm-thick $\mathrm{Co}_{1-y} \mathrm{O}$ layers are shown in Fig. 1(c) and 1(d) grown at oxygen pressures of $p\left(\mathrm{O}_{2}\right)=3.3 \times 10^{-7} \mathrm{mbar}$ and $1.0 \times 10^{-5}$ mbar, respectively. All diffraction patterns from the AFM layers show a transmission image, i.e., diffraction from a rough surface with islands. ${ }^{22}$ In order to explain the observed RHEED patterns a (111) orientation of fcc $\mathrm{Co}_{1-y} \mathrm{O}$ is assumed. The calculated diffraction patterns are shown in Fig. 1(e). The fille circles represent the reciprocal lattice points of the undiluted $\mathrm{CoO}$ fcc lattice.

In the $0^{\circ}$ direction, the calculated pattern of fille circles [Fig. 1(e)] fit to the RHEED image of the $\mathrm{Co}_{1-y} \mathrm{O}$ layer prepared at low oxygen pressure [Fig. 1(c), left panel]. For the samples prepared at higher oxygen pressures, additional diffraction spots [open circles in Figs. 1(e) and 1(f)] appear at half the distance between the fille circles showing an additional structure with approximately twice the lattice constant in real space. We believe that these additional spots are due to the formation of $\mathrm{Co}_{3} \mathrm{O}_{4}$ upon dilution, which is also consistent with results from x-ray diffraction (see below). We conclude that almost defect-free $\mathrm{CoO}$ is deposited at low oxygen pressure $\left[p\left(\mathrm{O}_{2}\right)=3.3 \times 10^{-7} \mathrm{mbar}\right]$, while for higher oxygen pressures Co-deficien $\mathrm{Co}_{1-y} \mathrm{O}$ is formed. To further investigate the formation of the additional phase with 
(a)

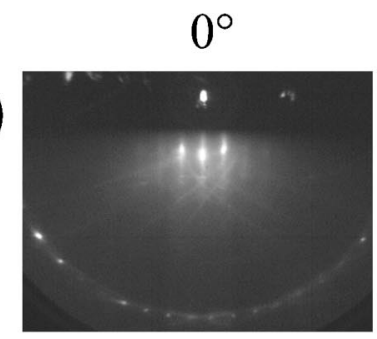

(b)

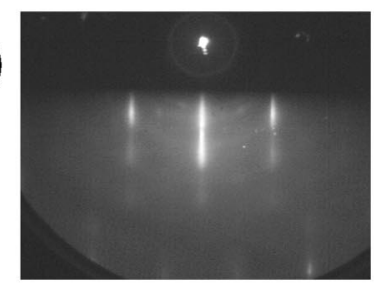

(c)

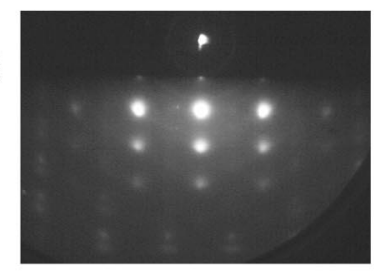

(d)

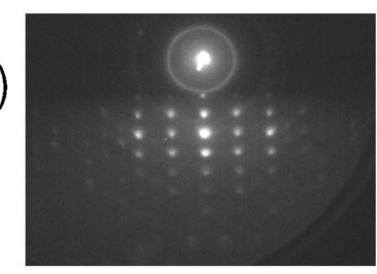

(e)

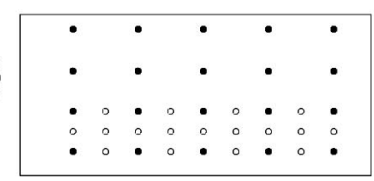

(f)

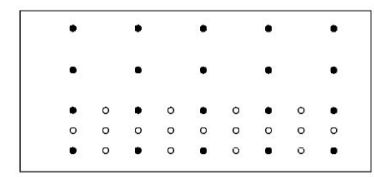

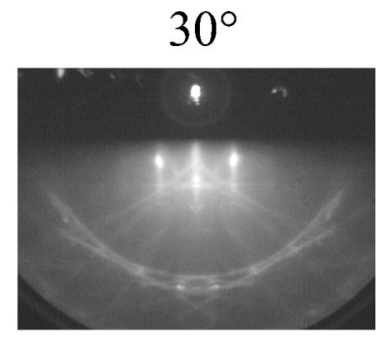
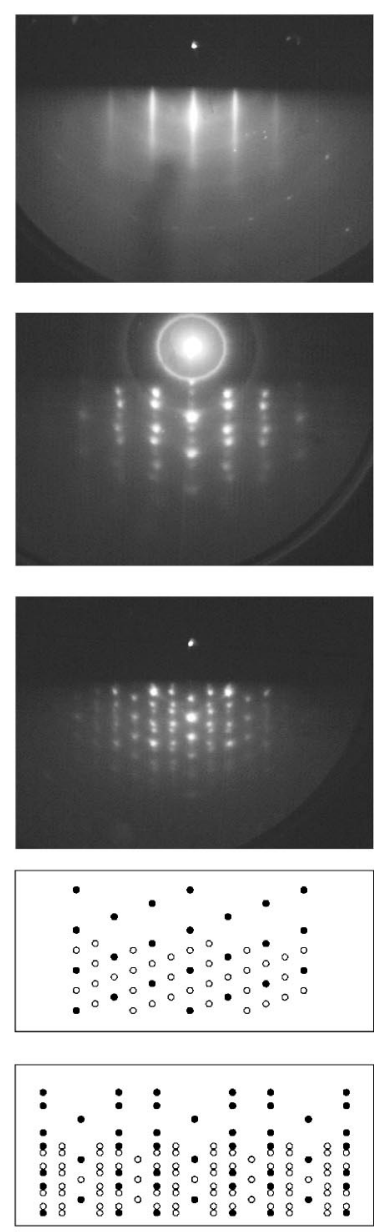

FIG. 1. RHEED images of the (a) (0001)-oriented sapphire substrate, (b) 6-nm Co layer on sapphire, and two 20-nm $\mathrm{Co}_{1-y} \mathrm{O}$ layers prepared at (c) $p\left(\mathrm{O}_{2}\right)=3.3 \times 10^{-7}$ mbar and at (d) $p\left(\mathrm{O}_{2}\right)$ $=1.0 \times 10^{-5}$ mbar on a 6-nm-thick Co layer. (e) and (f): Simulated reflection of the diffraction patterns of $\mathrm{Co}_{1-y} \mathrm{O}$ (111); (e) without twins and (f) with $60^{\circ}$ in-plane twins. Only solid dots fulfil the diffraction condition for the $\mathrm{CoO}$ fcc lattice. The two vertical panels show the patterns for $0^{\circ}$ and $30^{\circ}$ in-plane orientation of the incident

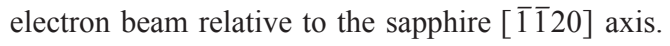

increasing oxygen pressure, we analyze the evolution of the RHEED intensity of the $(0,1 / 2)$ spot [open circles in Figs. $1(\mathrm{e})$ and 1(f)] as a function of oxygen pressure. Figure 2(a) shows a line scan through the RHEED diffraction pattern of $\mathrm{Co}_{1-y} \mathrm{O}$ prepared at high oxygen pressure $\left[p\left(\mathrm{O}_{2}\right)=1\right.$ $\times 10^{-5}$ mbar $]$. The $(0,1)$ peak corresponds to the undiluted fcc lattice of $\mathrm{CoO}$, while the $(0,1 / 2)$ peak appears for $\mathrm{Co}-$ deficien $\mathrm{Co}_{1-y} \mathrm{O}$. The relative intensity of the $(0,1 / 2)$ and $(0,1)$ peaks is strongly increasing as a function of oxygen
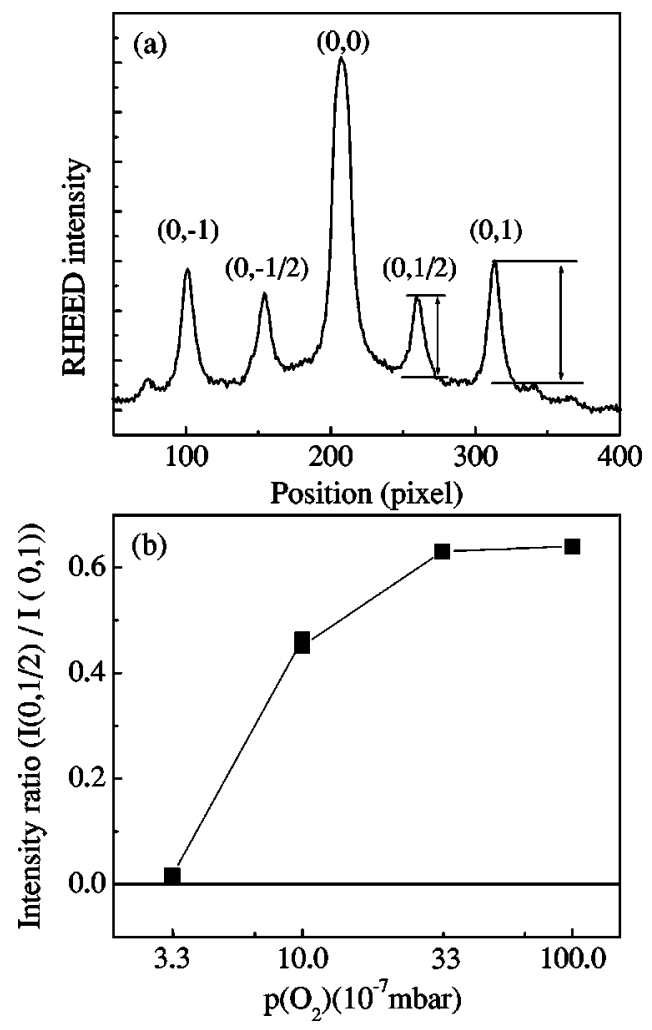

FIG. 2. (a) Line scan of the RHEED image shown in Fig. 1(d), left panel, for a $\mathrm{Co}_{1-y} \mathrm{O}$ layer prepared at $p\left(\mathrm{O}_{2}\right)=1.0$ $\times 10^{-5}$ mbar. (b) Dilution dependence of intensity ratio between the additional $(0,1 / 2)$ spot for overoxidized $\mathrm{Co}_{1-y} \mathrm{O}$ and the $(0,1)$ reflection for the $\mathrm{CoO}$ fcc structure.

pressure as seen in Fig. 2(b). This supports the notion that the number of volume defects in the AFM layer can be controlled by the oxygen pressure during deposition, i.e., the number of defects is continuously increasing with increasing oxygen pressure.

We now discuss the RHEED patterns along the $30^{\circ}$ direction in Fig. 1 (right panels). The calculated RHEED pattern in Fig. 1(e) does not reproduce the double spot structures as observed for all oxygen concentrations [see Figs. 1(c) and $1(\mathrm{~d})$, right panels]. In order to explain these diffraction patterns, we furthermore have to assume that $\mathrm{Co}_{1-y} \mathrm{O}$ grows in a twinned structure where crystallites are oriented $60^{\circ}$ relative to each other [compare Fig. 1(f) with Figs. 1(c) and 1(d), right panels]. Similar to the $0^{\circ}$ direction the undiluted sample [Fig. 1(c)] only shows reflection from a $\mathrm{CoO}$ fcc lattice, while the diluted samples also show reflection from the defect phase.

The main experimental features of the RHEED investigation of the $\mathrm{Co}_{1-y} \mathrm{O}$ layers are (i) the number of defects in $\mathrm{Co}_{1-y} \mathrm{O}$ continuously increases with increasing oxygen pressure during evaporation and (ii) the layers grow with $60^{\circ}$ twins. The same qualitative finding were observed in $\mathrm{Mg}$ diluted $\mathrm{CoO}$ layers (not shown). In the following we will refer to the samples with the lowest defect concentration $\left[p\left(\mathrm{O}_{2}\right)=3.3 \times 10^{-7}\right.$ mbar and $\left.x(\mathrm{Mg})=0.0\right]$ as unintentionally diluted.

Additional structural characterization was carried out by 


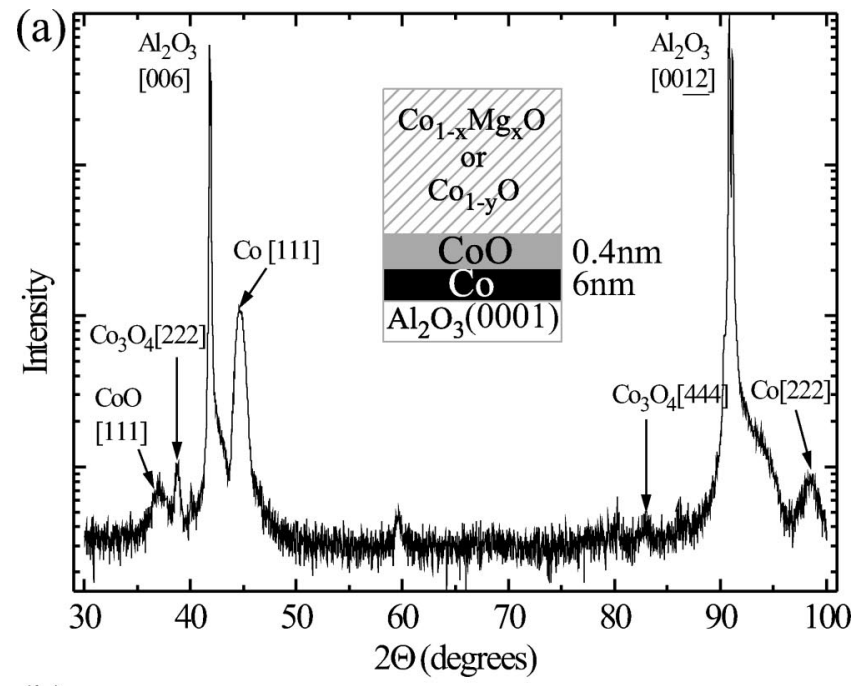

(b)

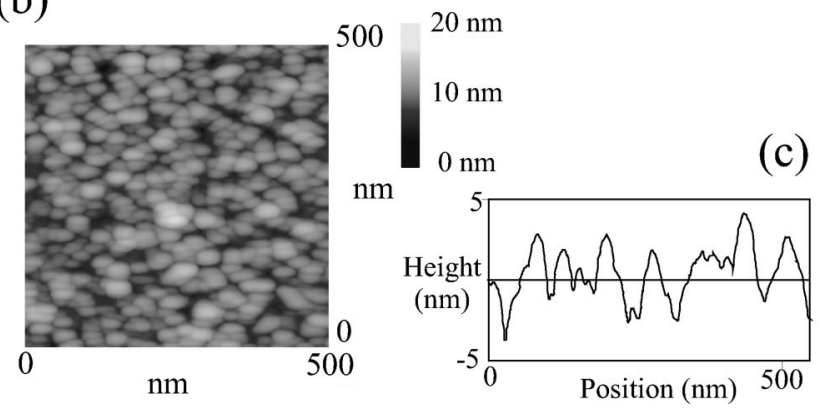

FIG. 3. (a) High-angle $x$-ray diffraction $(\lambda=0.15418 \mathrm{~nm})$ for $\mathrm{Co} / \mathrm{Co}_{1-y} \mathrm{O}$ with $\mathrm{Co}_{1-y} \mathrm{O}$ prepared at $p\left(\mathrm{O}_{2}\right)=3 \times 10^{-6}$ mbar. The sample structure is schematically depicted in the inset. (b) Atomic force microscopy image of a 20 -nm-thick $\mathrm{Co}_{1-y} \mathrm{O}$ layer prepared at $p\left(\mathrm{O}_{2}\right)=3 \times 10^{-6}$ mbar layer on a 6-nm-thick Co layer. Crystallite sizes range between 25 and $35 \mathrm{~nm}$. (c) Line scan of image.

ex situ $\mathrm{x}$-ray diffraction using $\mathrm{Cu} K_{\alpha}$ radiation $(\lambda$ $=0.15418 \mathrm{~nm}$ ). A high-angle $\theta-2 \theta$ scan of a $\mathrm{Co} / \mathrm{Co}_{1-y} \mathrm{O}$ bilayer with the antiferromagnet prepared at $p\left(\mathrm{O}_{2}\right)$ $=3 \times 10^{-6}$ mbar is shown in Fig. 3(a). Besides prominent $\left[\begin{array}{lll}0 & 0 & l\end{array}\right]-\mathrm{Al}_{2} \mathrm{O}_{3}$ substrate peaks, only $\left[\begin{array}{lll}1 & 1 & 1\end{array}\right]$ reflection and those of higher order are seen for both $\mathrm{Co}$ and $\mathrm{CoO}$, which is consistent with the RHEED results. In addition, [ $\left.\begin{array}{lll}1 & 1 & 1\end{array}\right]$-oriented $\mathrm{Co}_{3} \mathrm{O}_{4}$ is observed.

To further investigate the surface of the $\mathrm{Co}_{1-y} \mathrm{O}$ layers, ex situ atomic force microscopy images were taken. Figure 3(b) shows an atomic force microscope image for a 20 -nm-thick $\mathrm{Co}_{1-y} \mathrm{O}$ layer prepared at $p\left(\mathrm{O}_{2}\right)=3 \times 10^{-6}$ mbar on top of a 6-nm Co layer. As expected from the RHEED investigations a rough surface is found. The crystallite size ranges between $25 \mathrm{~nm}$ and $35 \mathrm{~nm}$. As is seen from the line scan in Fig. 3(c), the surface has a peak-to-peak height variation of approximately $6 \mathrm{~nm}$.

\section{MAGNETIC PROPERTIES}

The magnetic characterization of the samples was performed using a superconducting quantum interference device (SQUID) magnetometer. The samples were cooled from $320 \mathrm{~K}$, i.e., from above the Néel temperature $T_{N}(\mathrm{CoO})$

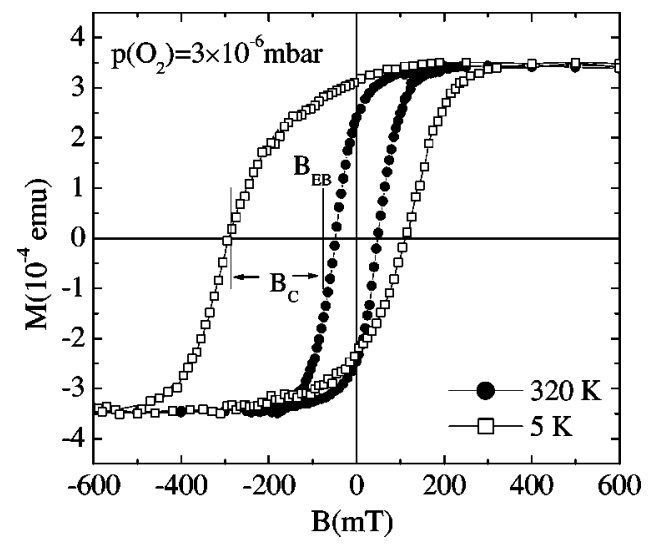

FIG. 4. Hysteresis loops of $\mathrm{Co}_{1-y} \mathrm{O} / \mathrm{Co} / \mathrm{Al}_{2} \mathrm{O}_{3}$ (0001) at $T$ $=5 \mathrm{~K}$ and $T=320 \mathrm{~K}$ with the $\mathrm{Co}_{1-y} \mathrm{O}$ prepared at $p\left(\mathrm{O}_{2}\right)=3$ $\times 10^{-6}$ mbar. The exchange bias fiel $B_{\mathrm{EB}}$ and the coercivity fiel $\mathrm{B}_{C}$ are indicated in the figure

$=291 \mathrm{~K}$ to $5 \mathrm{~K}$ in the presence of an external magnetic fiel $+B_{\mathrm{FC}}$, oriented parallel to the plane of the film Except for the explicit studies of the cooling fiel dependence (see Sec. IV E), a cooling fiel of $B_{\mathrm{FC}}=5 \mathrm{~T}$ was chosen for all measurements presented. This value is larger than the saturation fiel of the ferromagnetic Co layer.

\section{A. Hysteresis}

Figure 4 shows typical hysteresis loops above ( $T$ $=320 \mathrm{~K})$ and below $(T=5 \mathrm{~K})$ the Néel temperature of a sample with the $\mathrm{Co}_{1-y} \mathrm{O}$ layer grown at $p\left(\mathrm{O}_{2}\right)$ $=3 \times 10^{-6}$ mbar. The loop at low temperature exhibits a large exchange bias shift $B_{\mathrm{EB}}$ towards negative magnetic fields which is opposite to the cooling fiel direction. In addition, a strong increase in the coercivity fiel $B_{C}$ is observed at $5 \mathrm{~K}$ compared to $320 \mathrm{~K}$ leading to a significan broadening of the width of the loops.

Like most conventional magnetization probes, SQUID magnetometry is not layer or element specifi but rather measures the whole FM/AFM bilayer magnetization. In addition to the magnetization of the FM layer, both interface and volume magnetization of the diluted AFM layer will therefore contribute to the total magnetization. If the magnetization of the antiferromagnet is irreversible under fiel reversal, it can be identifie as a vertical shift of the measured hysteresis loop. Such an AFM magnetization was firs observed in hysteresis loops of $\mathrm{Fe} / \mathrm{FeF}_{2}$ and $\mathrm{Fe} / \mathrm{MnF}_{2} \cdot{ }^{23}$ Both, positive and negative vertical shifts were found and attributed to positive (ferromagnetic) and negative (antiferromagnetic) FM/AFM interface coupling, respectively. However, the origin of the induced moment could not directly be assigned to either interface or volume magnetization.

To investigate the change of the AFM magnetization with the number of introduced volume defects in the AFM layer, we performed high accuracy magnetization measurements of the vertical magnetization shift for both unintentionally diluted and oxygen-diluted samples grown at $p\left(\mathrm{O}_{2}\right)$ $=3.3 \times 10^{-7}$ mbar and $p\left(\mathrm{O}_{2}\right)=3 \times 10^{-6}$ mbar, respectively. The shift was determined at $T=20 \mathrm{~K}$ and is given by 


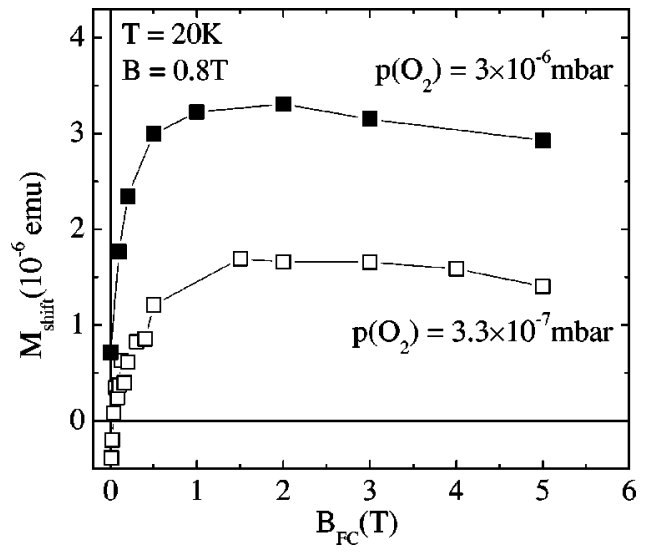

FIG. 5. Vertical magnetization shift $M_{\text {shift }}$ vs cooling fiel $B_{\mathrm{FC}}$ for $\mathrm{Co} / \mathrm{Co}_{1-y} \mathrm{O}$ samples with $\mathrm{Co}_{1-y} \mathrm{O}$ prepared at different oxygen pressures. Data are taken at $B= \pm 0.8 \mathrm{~T}$ and $T=20 \mathrm{~K}$ and are extracted as described in the text.

$M_{\text {shift }}=\left|M\left(B_{+}\right)\right|-\left|M\left(B_{-}\right)\right|$, where $B_{+}$and $B_{-}$are chosen so that the FM layer is fully saturated with $\left|B_{+}\right|=\left|B_{-}\right|$. The data shown in Fig. 5 were taken at $B_{ \pm}= \pm 0.8$ T. As is seen in Fig. 5, at large cooling field $M_{\text {shift }}$ is positive and overall increasing with dilution of the AFM layer at all cooling fields This increase can directly be linked to the creation of additional volume defects in the AFM layer as shown by the above RHEED analysis. It further supports that a domain state is developed in the antiferromagnet after fiel cooling carrying a surplus magnetization, which increases with dilution. It is important to note that in our experiments we measure the total AFM surplus magnetization as was also investigated by similar magnetization probes in $\mathrm{CoO} / \mathrm{MgO}$ multilayers ${ }^{24}$ and $\mathrm{Co}_{x} \mathrm{Mg}_{1-x} \mathrm{O}$ powder samples. ${ }^{25}$ Although, this surplus magnetization does not equal the irreversible domain state (IDS) magnetization $m_{\text {IDS }}$ of the AFM interface layer define in Sec. IV A of Ref. 7, we fin striking qualitative agreement that the $\mathrm{EB}$ fiel indeed is proportional to the measured AFM magnetization (see the next section and Sec. IV E).

In the MC simulations, ${ }^{7}$ contributions from both interface and bulk magnetization of the AFM layer can be separated. After zero-fiel cooling, the bulk of the AFM layer does not carry surplus magnetization (see Fig. 5 in Ref. 7), while it should dominate the total surplus magnetization for larger cooling fields Indeed, we observe a strong reduction of $M_{\text {shift }}$ for cooling field below $1 \mathrm{~T}$ as is seen in Fig. 5. Thus, it is suggestive that the low-fiel AFM magnetization primarily originates from the AFM spins close to the interface. While for diluted samples, $M_{\text {shift }}$ remains finit and positive for zero cooling field it changes sign for unintentionally diluted samples. The former case is consistent with positive (ferromagnetic) $\mathrm{FM} / \mathrm{AFM}$ interface coupling in $\mathrm{Co} / \mathrm{CoO}$ (compare with AFM magnetization loops in MC simulations as shown in Fig. 4 of Ref. 7). This conclusion is also consistent with the cooling fiel dependence of the EB shift which will be discussed in Sec. IVE.

However, it has to be reemphasized that by SQUID magnetometry we do not only measure the IDS magnetization $m_{\text {IDS }}$ of the AFM interface layer, which is responsible for the
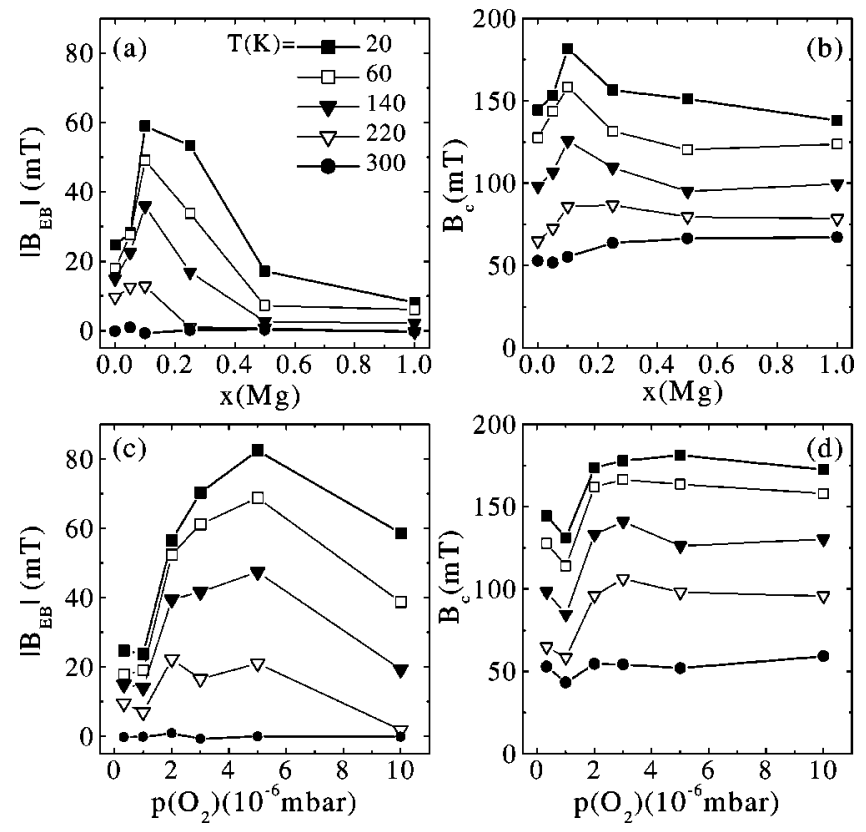

FIG. 6. (a) EB fiel and (b) coercivity fiel vs Mg concentration $x$ in the $\mathrm{Co}_{1-x} \mathrm{Mg}_{x} \mathrm{O}$ layer for various temperatures. (c) $\mathrm{EB}$ fiel and (d) coercivity fiel vs oxygen pressure during deposition of the $\mathrm{Co}_{1-y} \mathrm{O}$ layer at the same temperatures. Note that only absolute EB values are plotted. All lines are guides to the eye.

EB coupling in the DS model. Therefore, at present it is not clear as to how much the sign reversal of $M_{\text {shift }}$ at $B_{\mathrm{FC}}=0$ is related to the EB coupling.

\section{B. Influenc of dilution}

The dilution dependence of both the EB fiel $\left|B_{\mathrm{EB}}\right|$ and coercivity fiel $B_{C}$ for $\mathrm{Mg}$-diluted $\mathrm{Co}_{1-x} \mathrm{Mg}_{x} \mathrm{O}$ samples is shown in the upper panels of Fig. 6, while in the lower panels of Fig. 6 analogous results of Co-deficien $\mathrm{Co} / \mathrm{Co}_{1-y} \mathrm{O}$ samples are depicted. The EB is enhanced by a factor of 3 to 4 for both types of defects in the AFM layer. Maximum enhancement is obtained for $x(\mathrm{Mg})=0.1$ and $p\left(\mathrm{O}_{2}\right)=5 \times 10^{-6}$ mbar. Note that compared to our previous work, ${ }^{6}$ we prepared additional Co-deficien samples at an oxygen pressure of $p\left(\mathrm{O}_{2}\right)=5 \times 10^{-6} \mathrm{mbar}$, which show the largest EB fiel of all samples. Within the DS model, the observed increase of the EB shift with an increasing number of defects can be related to the formation of volume domain walls, which preferentially pass through the nonmagnetic defects at no cost of exchange energy. This leads to the experimentally observed excess magnetization of the antiferromagnet. The increase of excess magnetization between unintentionally diluted samples $\left[x(\mathrm{Mg})=0, p\left(\mathrm{O}_{2}\right)=3.3\right.$ $\left.\times 10^{-7} \mathrm{mbar}\right]$ and optimally diluted samples $[x(\mathrm{Mg})=0.1$ or $\left.p\left(\mathrm{O}_{2}\right)=5 \times 10^{-6} \mathrm{mbar}\right]$ is directly verifie by the vertical magnetization shift of the hysteresis loop (see Sec. IV A). This strongly supports the fact that the domain state in the AFM layer as well as the EB effect is caused and controlled by the defects. For large dilution $\left[x(\mathrm{Mg})>0.25 ; p\left(\mathrm{O}_{2}\right)>5\right.$ $\left.\times 10^{-6} \mathrm{mbar}\right]$ the EB again decreases as the antiferromagnetic order is increasingly suppressed and eventually the 
connectivity in the AFM lattice is lost. Residual EB at high dilutions $[x(\mathrm{Mg})=1.0]$ has to be attributed to the $0.4-\mathrm{nm}$ $\mathrm{CoO}$ interface layer and the underlying oxidized layer (see also Sec. IV C). A similar behavior is observed in other EB systems consisting of diluted metallic antiferromagnets. ${ }^{26-28}$ It has also be shown that the EB can be enhanced by ion irradiation of the whole FM/AFM sample. ${ }^{29}$

MC simulations (see Fig. 6 of Ref. 7) qualitatively reproduce both the initial increase of EB with increasing dilution and its decrease at larger dilutions. However, the concentrations for optimally diluted samples significantl differ between experiment $[x(\mathrm{Mg}) \approx 0.15]$ and theory $(p \approx 0.6)$. A possible origin of this difference is the presence of grain boundaries in the twinned AFM layer which reduce the domain-wall energy, thus leading to a finit EB without intentional dilution of the AFM layer as observed for the unintentionally diluted samples. This conclusion is consistent with the rather small EB found in untwinned and single crystalline antiferromagnets. ${ }^{30-32}$ As seen in Figs. 6(a) and 6(c) the defect concentration for maximum EB depends on the temperature, i.e., it shifts towards smaller values at elevated temperatures, which is also qualitatively observed in $\mathrm{MC}$ simulations (see Fig. 6 in Ref. 7).

We next discuss the dilution dependence of the coercivity fiel $B_{C}$, which is shown in Figs. 6(b) and 6(d). Similar to the EB field it changes nonmonotonically with dilution and shows maximum values at the same defect concentrations as the EB, although its relative changes are smaller than those observed in the EB field Like the EB, the coercivity also strongly decreases with increasing temperature, which is common for many EB systems. ${ }^{5}$ The change in coercivity with temperature is caused by the change in the coupling of the FM (Co) layer with the AFM ( $\mathrm{CoO})$ layer.

\section{Temperature dependence}

The temperature dependence of the exchange bias fiel $\left|B_{\mathrm{EB}}\right|$ for the $\mathrm{Mg}$-diluted $\mathrm{Co} / \mathrm{Co}_{1-x} \mathrm{Mg}_{x} \mathrm{O}$ samples is shown in Figs. 7(a)-7(e) at the same $\mathrm{Mg}$ concentrations as in Fig. 6(a). For all samples the EB fiel is monotonically increasing with decreasing temperature. It almost varies linearly with temperature near optimum dilution $[x(\mathrm{Mg})=0.1$ and $x(\mathrm{Mg})=0.25]$, while it saturates at low temperatures and low dilutions [Figs. 7(a) and 7(b)]. The former dependence agrees well with the temperature behavior as obtained by MC simulations near optimum dilution (see Fig. 8 in Ref. 7).

The results are described by the DS model as follows: A metastable domain state is frozen at low temperatures after fiel cooling, which inhibits domain-wall motion. Thermally activated domain-wall motion becomes more favorable at elevated temperatures, which leads to a reduction of $m_{\mathrm{IDS}}$ and thus to a decrease of the exchange bias fiel as observed experimentally.

The EB vanishes above the so-called blocking temperature $T_{B}$, which might significantl differ from the Néel temperature $T_{N}$ of the antiferromagnet. ${ }^{7,33}$ Its monotonic decrease with increasing $\mathrm{Mg}$ dilution in $\mathrm{Co} / \mathrm{Co}_{1-x} \mathrm{Mg}_{x} \mathrm{O}$ samples can be seen by the arrows in Figs. 7(a)-7(e) and is also plotted in Fig. 8. We observe a similar but weaker de-
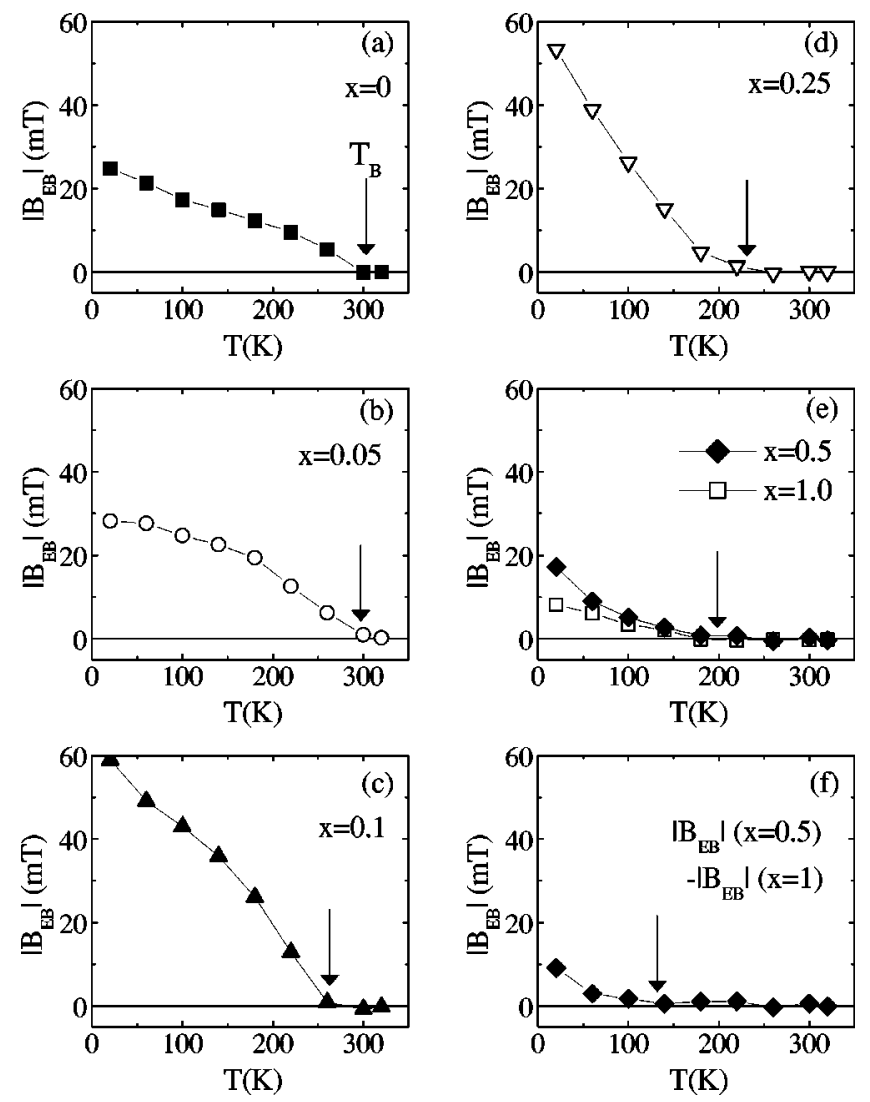

FIG. 7. (a)-(e) Temperature dependence of EB fiel for the Mg-diluted $\mathrm{Co} / \mathrm{Co}_{1-x} \mathrm{Mg}_{x} \mathrm{O}$ samples with $x=0,0.05,0.1,0.25,0.5$, and 1.0 as shown in Fig. 6(a). (f) Difference in EB field for samples with $x=0.5$ and $x=1.0$. The blocking temperature is marked by an arrow in each figure

crease of $T_{B}$ for the $\mathrm{Co} / \mathrm{Co}_{1-y} \mathrm{O}$ samples (not shown). Note that the blocking temperature is not completely diminished for the fully diluted sample with $x(\mathrm{Mg})=1.0$. As already discussed above, we attribute this remaining EB coupling to

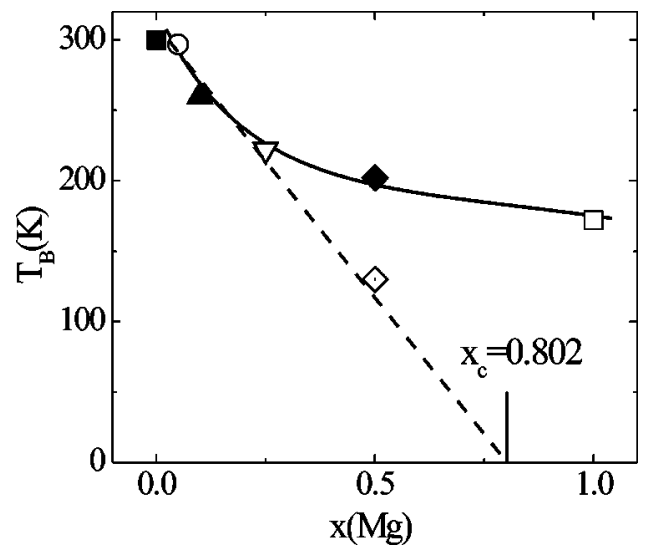

FIG. 8. Dilution dependence of blocking temperature $T_{B}$ for the $\mathrm{Mg}$ diluted $\mathrm{Co} / \mathrm{Co}_{1-x} \mathrm{Mg}_{x} \mathrm{O}$ samples as shown in Fig. 7. For $x$ $=0.5$ the lower $T_{B}$ (dot-center diamond) is taken from Fig. 7(f). All lines are guides to the eye. The percolation threshold $x_{c}$ for a threedimensional fcc lattice with nearest neighbor interaction is included. 


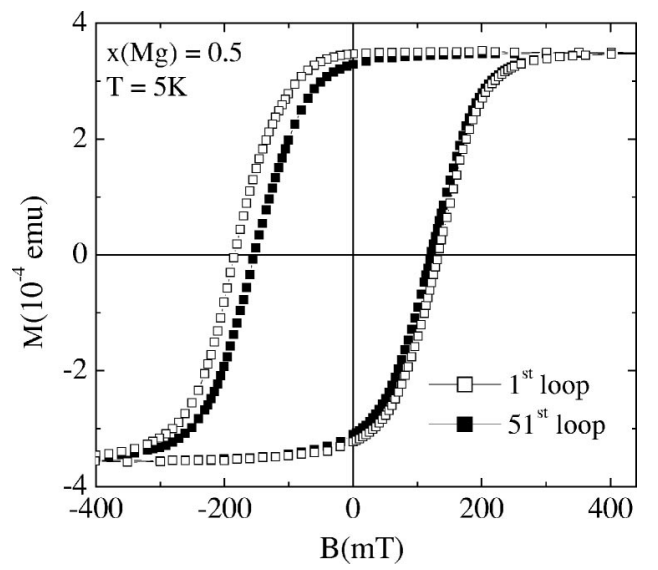

FIG. 9. Training effect of $\mathrm{Co} / \mathrm{Co}_{1-x} \mathrm{Mg}_{x} \mathrm{O}$ sample with $x=0.5$. Plotted are the firs and the 51 st hysteresis loops at $T=5 \mathrm{~K}$ after fiel cooling.

the $\mathrm{CoO}$ interface layer and the underlying oxidized layer. This residual EB coupling is likely also to be relevant for the temperature dependence of the EB fiel for the sample with $x(\mathrm{Mg})=0.5$, as can be seen in Fig. 7(e). To further separate the contributions to the EB coupling from the undiluted interface layer from that of the diluted AFM volume layer, we subtract the EB field for the sample with $x(\mathrm{Mg})=1.0$ from the sample with $x(\mathrm{Mg})=0.5$, which is depicted in Fig. 7(f). The resulting blocking temperature, which is strongly reduced, is also included in Fig. 8 at $x(\mathrm{Mg})=0.5$. Note that all other samples do not show any significan change in blocking temperature when performing a similar subtraction (not shown).

As is seen in Fig. 8, the modifie blocking temperatures linearly decrease with increasing Mg dilution of the AFM volume layer (dashed line). In particular, the extrapolated dilution, above which EB coupling vanishes completely $\left(T_{B} \rightarrow 0\right)$, is close to the percolation threshold $\left(x_{c}=0.802\right)$ for antiferromagnetism in a three-dimensional fcc lattice with nearest-neighbor interaction. ${ }^{34}$ This supports the notion that no global EB coupling remains once the connectivity of the AFM spin lattice is lost.

\section{Training effect}

Most thin-fil EB systems show a reduction of the EB shift upon subsequent magnetization reversals of the FM layer, ${ }^{5,35,36}$ which is the so-called training effect. For all Mgdiluted $\mathrm{Co} / \mathrm{Co}_{1-x} \mathrm{Mg}_{x} \mathrm{O}$ samples we measured the training effect at $T=5 \mathrm{~K}$ after fiel cooling in $B_{\mathrm{FC}}=5 \mathrm{~T}$. Typical magnetization reversals corresponding to the firs and 51st hysteresis loops are shown in Fig. 9 for the sample with $x(\mathrm{Mg})=0.5$. Besides a clear, but rather small reduction of the exchange bias shift, a decrease of the coercivity fiel is observed. The training effect implies that during magnetization reversal the FM layer does not reverse homogeneously nor reversibly. According to the DS model, the training effect is due to a rearrangement of the AFM domain structure, which results in a partial loss of the IDS magnetization $m_{\text {IDS }}$ of the AFM interface layer during fiel cycling. ${ }^{7}$ This magnetization loss leads to a reduction of the EB shift.
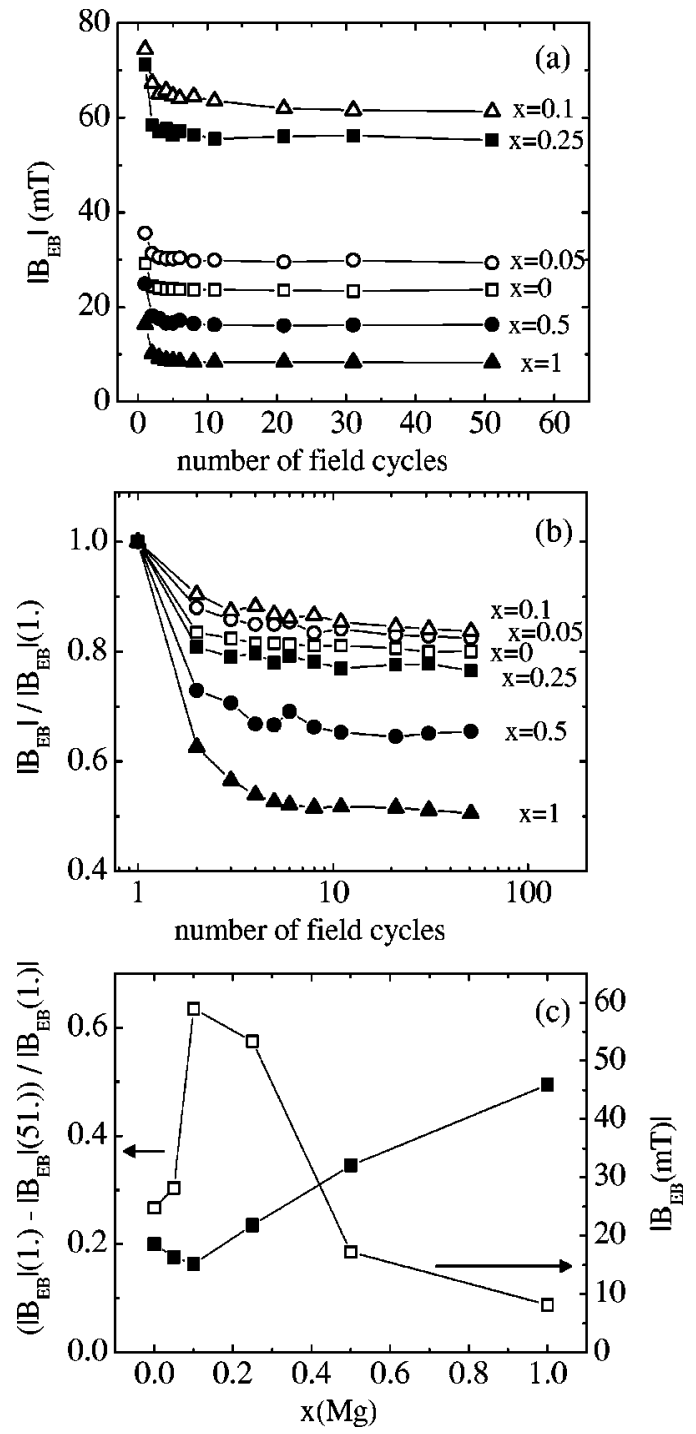

FIG. 10. Dilution dependence of the training effect for $\mathrm{Co} / \mathrm{Co}_{1-x} \mathrm{Mg}_{x} \mathrm{O}$ samples. (a) EB shift as a function of subsequent hysteresis cycles for different $\mathrm{Mg}$ concentrations $x$ at $T=5 \mathrm{~K}$. (b) Normalized EB values from (a) plotted on a semilog scale. Note that there is almost no training effect after ten fiel cycles. (c) Relative training effect vs $\mathrm{Mg}$ concentration (fille squares) superimposed with absolute EB shift at $T=5 \mathrm{~K}$ from Fig. 6(a).

To further investigate the relevance of the AFM domain structure on the training effect, we focus on its dilution dependence for the Mg-diluted samples (as shown in Fig. 6), which is depicted in Fig. 10 at $T=5 \mathrm{~K}$. Independent of the dilution, the EB shift is strongly reduced only during the firs fiel cycles and then remains almost constant. This behavior is in striking qualitative agreement with MC simulations (see Fig. 9 of Ref. 7).

To further explore the magnitude of the training effect, we plotted the relative decrease of the EB shift by normalizing the data from Fig. 10(a) by their initial value at each dilution. As is seen in Fig. 10(b), the magnitude of this relative training effect behaves nonmonotonically with dilution, with the smallest effect observed for samples near optimum dilution $[x(\mathrm{Mg})=0.1]$. In order to more easily visualize the dilution 
dependence, we plotted the relative training effect between the firs and 51st fiel cycles as a function of $\mathrm{Mg}$ dilutions in Fig. 10(c) as its asymptotic limes together with the absolute EB fiel values at $T=5 \mathrm{~K}$. We fin that both EB shift and magnitude of the training effect are closely related in their dilution dependence. In other words, at optimum dilution the exchange bias is strongest with the smallest training effect, while at high and low dilution the exchange bias decreases while the training effect is increased.

This supports the notion that the formation of volume domains in the AFM layer plays a crucial role in the exchange bias interaction at the FM/AFM interface. The observed dilution dependence of the training effect can be interpreted within the DS model as follows. Magnetization reversal of the FM layer causes irreversible changes in the AFM domain structure, which yield a reduction of the IDS magnetization $m_{\mathrm{IDS}}$ of the AFM interface layer and thus a drop in the EB shift. Energetically, AFM domain walls are most strongly pinned near optimum dilution. This pinning results in large energy barriers and prohibits domain-wall motion upon FM magnetization reversal leading to a small training effect. At larger dilutions the size and the connectivity of the AFM spin lattice gets reduced. This results in a decrease of the AFM domain-wall barrier and a decrease of EB. At the same time AFM domain-wall motion and relaxation become easier upon FM magnetization reversal leading to an enhancement of the training effect.

\section{E. Cooling fiel dependence}

The exchange bias effect reveals a striking dependence on the magnitude of the cooling fiel $B_{\mathrm{FC}}$. At large cooling fields the EB is (i) either constant, ${ }^{37}$ or moderately reduced in most EB systems, or (ii) changes its sign (positive exchange bias) in systems such as $\mathrm{Fe} / \mathrm{FeFe}_{2}$ and $\mathrm{Fe} / \mathrm{MnFe}_{2}{ }^{38,39}$ These results were explained by antiferromagnetic interface coupling between ferromagnet and antiferromagnet. In the MC simulations, both types of dependencies are qualitatively obtained and can be linked to the sign of the interface coupling. ${ }^{7}$ While the former case (i) is obtained for positive (ferromagnetic) FM/AFM interface coupling, a negative (antiferromagnetic) interface coupling yields the latter case (ii).

To investigate the sign of the interface coupling in $\mathrm{Co} /$ $\mathrm{CoO}$ and its dependence on the AFM volume dilution, we studied the cooling fiel dependence of the same $\mathrm{Co} / \mathrm{Co}_{1-y} \mathrm{O}$ samples as shown in Fig. 6(c) at both low $\left[p\left(\mathrm{O}_{2}\right)=3.3\right.$ $\times 10^{-7}$ mbar $]$ and optimum $\left[p\left(\mathrm{O}_{2}\right)=5 \times 10^{-6} \mathrm{mbar}\right] \mathrm{de}-$ fect concentrations. For all cooling field the FM layer was firs magnetized at a fiel of $5 \mathrm{~T}$ and a temperature of $T$ $=320 \mathrm{~K}$. Then it was cooled to $T=20 \mathrm{~K}$ for cooling field between $B_{\mathrm{FC}}=0 \mathrm{~T}$ and $5 \mathrm{~T}$. As is shown in Fig. 11, the EB increases at low cooling field while it slightly decreases at larger field for both samples. The initial increase we primarily attribute to the magnetization of the FM layer, which is not fully saturated during fiel cooling at low cooling field as can be seen in the typical hysteresis loops for the moderately diluted samples at $T=320 \mathrm{~K}$ (see Fig. 4). Since the
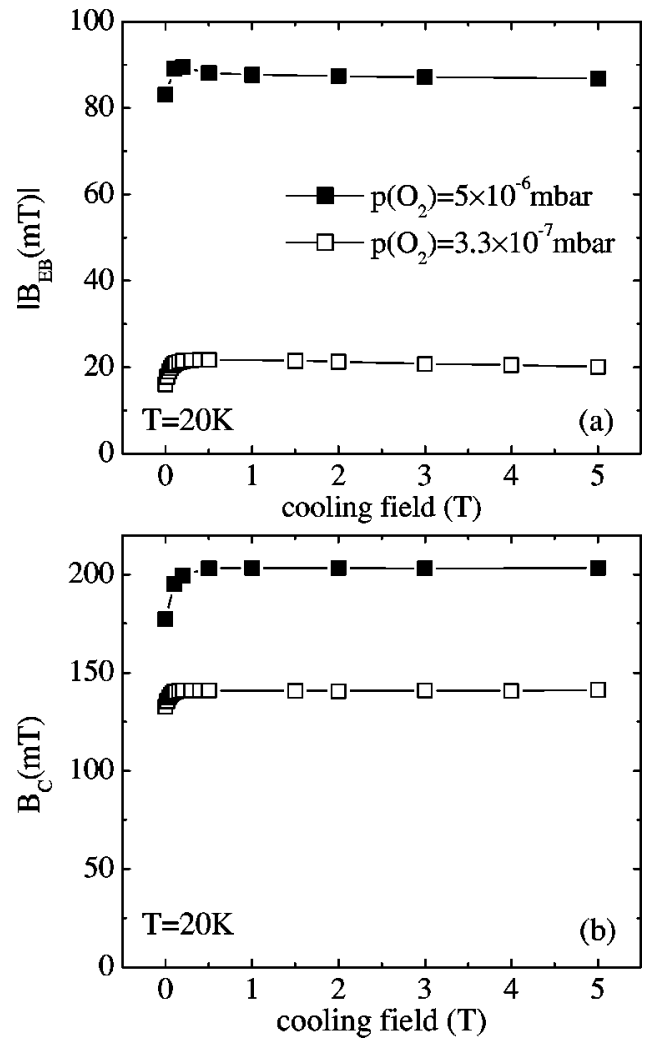

FIG. 11. (a) EB fiel and (b) coercivity fiel vs cooling fiel for $\mathrm{Co} / \mathrm{Co}_{1-y} \mathrm{O}$ samples at $T=20 \mathrm{~K}$ with the $\mathrm{Co}_{1-y} \mathrm{O}$ layer prepared at different oxygen pressures.

FM layer magnetization determines the global exchange bias ${ }^{40}$ its decrease towards small field will reduce the EB coupling.

Because of the only slight decrease of the EB at high cooling field and no observation of positive EB we conclude that we have positive (ferromagnetic) interface coupling between $\mathrm{Co}$ and $\mathrm{CoO}$. This is consistent with no change in sign of $B_{\mathrm{EB}}$ in $\mathrm{NiFe} / \mathrm{CoO}$ up to $B_{\mathrm{FC}}=5 \mathrm{~T} .{ }^{37}$ This conclusion is further confirme by the observation of a positive vertical shift of the hysteresis loops (see Sec. IV A). The Monte Carlo simulations show qualitatively the same EB dependence on the cooling fiel as observed here for positive interface coupling (see Fig. 10 in Ref. 7). For comparison we plotted the corresponding coercive field in Fig. 11(b). The same qualitative behavior is observed at low cooling field as in Fig. 11(a) for $B_{\mathrm{EB}}$, while the coercive fiel remains constant at larger cooling fields

\section{F. AFM layer thickness}

In the literature there exists no clear statement about the dependence of the exchange bias on the AFM layer thickness. Agreement seems to exist that there has to be a minimum AFM thickness in order to yield EB. For larger thicknesses the observations can be classifie into two characteristic types of dependencies. ${ }^{41,42}$ (i) For thicknesses larger than a minimum value, the EB remains constant as a function of the AFM layer thickness. (ii) With increasing AFM layer thickness the EB fiel goes through a maximum 

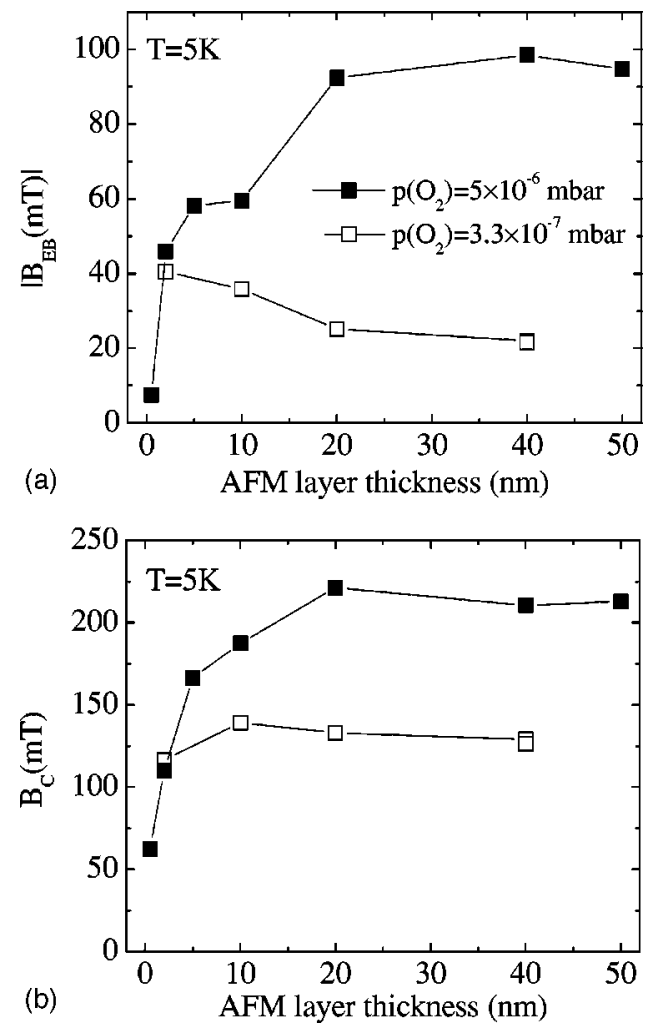

FIG. 12. (a) EB fiel and (b) coercivity fiel as a function of the AFM layer thickness in $\mathrm{Co} / \mathrm{Co}_{1-y} \mathrm{O}$ samples for both unintentionally diluted and optimally diluted $\mathrm{Co}_{1-y} \mathrm{O}$ layers prepared at oxygen pressures of $p\left(\mathrm{O}_{2}\right)=3.3 \times 10^{-7}$ mbar and $p\left(\mathrm{O}_{2}\right)=5.0$ $\times 10^{-6}$ mbar, respectively.

and then continuously decreases. The AFM layer thickness at which maximum EB occurs or beyond which EB saturates strongly depends on the particular materials chosen as well as on their preparation conditions.

We therefore investigated the AFM layer thickness dependence of the EB fiel for both unintentionally diluted $\left[p\left(\mathrm{O}_{2}\right)=3.3 \times 10^{-7} \mathrm{mbar}\right]$ and optimally diluted $\left[p\left(\mathrm{O}_{2}\right)\right.$ $\left.=5 \times 10^{-6} \mathrm{mbar}\right] \mathrm{Co}_{1-y} \mathrm{O}$ layers, which is depicted in Fig. 12(a) at $T=5 \quad \mathrm{~K}$. For optimally diluted samples, the EB fiel strongly increases with increasing AFM layer thickness and saturates above $20 \mathrm{~nm}$, which roughly corresponds to the size of the AFM crystallites [see Sec. III and Fig. 3(b)]. The EB fiel for unintentionally diluted samples, however, is decreasing with increasing AFM layer thickness and levels off at large thickness. We note that just by varying the defect density in the volume of the AFM layer we observe both types of thickness dependencies as reported in other EB systems. The MC simulations reproduce this trend (see Fig. 12 in Ref. 7, and Ref. 43) qualitatively.

Within the DS model, these different dependencies are described in the following way. At small levels of disorder (unintentionally diluted samples), AFM domains can only be created at the cost of high energy. Since it is energetically unfavorable to close the domains parallel to the layers, the domain walls will extend through the whole thickness of the AFM layer perpendicular to the FM/AFM interface. For that case, the domain-wall energy increases proportionally to the
AFM layer thickness. ${ }^{11,12}$ Thus, the formation of a domain wall in the AFM layer becomes less favorable with increasing AFM layer thickness. This results in a reduction of both the number of domain walls and the IDS magnetization $m_{\mathrm{IDS}}$, thus leading to a drop in the EB fiel with increasing AFM layer thickness. For large AFM layer thicknesses, the low defect density in the volume of the AFM layer prohibits the formation of domain walls even if there is disorder at the interface. For very thin AFM layers, on the other hand, the disorder from the interface dominates and domain-wall formation is energetically favorable, which then leads to a large exchange bias field

For the optimally diluted samples, domain walls can be created in an external magnetic fiel at less of a cost of energy due to the nonmagnetic defects. The IDS magnetization $m_{\mathrm{IDS}}$ at the interface, which is responsible for the EB, is stabilized by the AFM volume domain structure. This is consistent with the results of the MC simulations. ${ }^{7}$ Hence, the domain structure becomes more and more stable with increasing AFM layer thickness leading to an increase of the exchange bias. Assuming that grain boundaries strongly lower the antiferromagnetic coupling strength, the grains may act magnetically independent, leading to a constant exchange bias for AFM thicknesses larger than the grain size. This is consistent with our experimental observations in Fig. 12.

\section{CONCLUSIONS}

In conclusion, we have tested and demonstrated experimentally that the theoretical DS model for exchange bias, ${ }^{7}$ inherently based on disorder in the volume of the AFM layer, gives consistent insight into the mechanism of the exchange bias effect. Most of the characteristic EB phenomena, such as the vertical shift of the hysteresis loop, dilution dependence, temperature dependence, training effect, cooling fiel dependence, and antiferromagnetic layer thickness dependence, fin a consistent description in the combination of structural disorder together with the formation of an antiferromagnetic domain state. The disorder can result from interfacial roughness, defects in the volume part of the AFM, grain boundaries, or from other sources.

The DS model can also account for the large variation of observed EB coupling constants even for identical FM/AFM material systems. ${ }^{5}$ Samples of the same FM/AFM material system prepared by different deposition and/or oxidization techniques on various substrates result in a large variation in crystalline, compositional, as well as interfacial properties. Our experimental studies corroborated by $\mathrm{MC}$ simulations of the DS model indicate that the AFM domain structure crucially depends on the particular configuratio and density of volume defects (such as deficiencie of magnetic atoms or ions) as well as on structural defects (such as grain boundaries, screw dislocations, twin boundaries, and others) in the AFM layer. This results in different spin configuration as well as a different irreversible domain state magnetization $m_{\text {IDS }}$ of the AFM interface layer, which cause a strong variation in the exchange bias coupling strength at the FM/AFM interface [see Figs. 6(a) and 6(c)]. As various fabrication 
techniques and preparation conditions crucially lead to different types of defects and defect concentrations, the AFM domain structure and thus the EB coupling constant appear to be strongly sample dependent. Therefore, for the sake of comparing different EB systems, at least a relative tendency of increasing or decreasing EB fiel with varying defect concentrations should be known if not a detailed, possibly quantitative analysis of defect concentrations.

Controversies about the dependence of EB on the FM/ AFM interface roughness $\mathrm{s}^{39,44-50}$ might also be accounted for by the DS model. Any change of interface roughness due to variations in the preparation parameters, such as growth or annealing temperature, most likely also results in a change of defect structure and domain configuratio in the AFM layer, although their mutual interrelation is presently not under- stood. This model and further more detailed models ${ }^{51}$ might eventually explain a variety of additional EB effects not addressed here, such as perpendicular coupling, rotational hysteresis, ${ }^{52}$ rotatable anisotropy, ${ }^{53}$ asymmetric magnetization reversals, ${ }^{54,55}$ and others.

\section{ACKNOWLEDGMENTS}

We acknowledge the support of Dr. C. D. Bredel who made high-resolution SQUID magnetometry available to us at the Technical University of Darmstadt. We thank M. Kuhlmann for performing the x-ray measurements as shown in Fig. 3. This work has been supported by the Deutsche Forschungsgemeinschaft through SFB Grant Nos. 341 and 491.
*Email address: bernd.beschoten@physik.rwth-aachen.de

†Email address: uli@thp.uni-duisburg.de

${ }^{1}$ W.H. Meiklejohn and C.P. Bean, Phys. Rev. 102, 1413 (1956).

${ }^{2}$ W.H. Meiklejohn and C.P. Bean, Phys. Rev. 105, 904 (1957).

${ }^{3}$ R.C.S. Kools, IEEE Trans. Magn. 32, 3165 (1996).

${ }^{4}$ Satoru Araki, Masashi Sano, Shuxiang Li, Yoshihiro Tsuchiya, Oliver Redon, Tetsuro Sasaki, Noriaki Ito, Kohichi Terunuma, Haruyuki Morita, and Mikio Matsuzaki, J. Appl. Phys. 87, 5377 (2000).

${ }^{5}$ For a recent review, see J. Nogués and Ivan K. Schuller, J. Magn. Magn. Mater. 192, 203 (1999).

${ }^{6}$ P. Miltényi, M. Gierlings, J. Keller, B. Beschoten, G. Güntherodt, U. Nowak, and K.D. Usadel, Phys. Rev. Lett. 84, 4224 (2000).

${ }^{7}$ U. Nowak, K.D. Usadel, J. Keller, P. Miltényi, B. Beschoten, and G. Güntherodt, preceding paper, Phys. Rev. B 66, 014430 (2002).

${ }^{8}$ W. Kleemann, Int. J. Mod. Phys. B 7, 2469 (1993).

${ }^{9}$ D. P. Belanger, in Spin Glasses and Random Fields, edited by A. P. Young (World Scientific Singapore, 1998).

${ }^{10}$ A.P. Malozemoff, Phys. Rev. B 35, 3679 (1987).

${ }^{11}$ A.P. Malozemoff, J. Appl. Phys. 63, 3874 (1988).

${ }^{12}$ A.P. Malozemoff, Phys. Rev. B 37, 7673 (1988).

${ }^{13}$ Pieter Kuiper, Barry G. Searle, Petra Rudolf, L.H. Tjeng, and C.T. Chen, Phys. Rev. Lett. 70, 1549 (1993).

${ }^{14}$ J. Stöhr, A. Scholl, T.J. Regan, S. Anders, J. Lüning, M.R. Scheinfein, H.A. Padmore, and R.L. White, Phys. Rev. Lett. 83, 1862 (1999).

${ }^{15}$ A. Scholl, J. Stöhr, J. Lüning, J.W. Seo, J. Fompeyrine, H. Siegwart, J.-P. Locquet, F. Nolting, S. Anders, E.E. Fullerton, M.R. Scheinfein, and H.A. Padmore, Science 287, 1014 (2000).

${ }^{16}$ F.U. Hillebrecht, H. Ohldag, N.B. Weber, C. Bethke, U. Mick, M. Weiss, and J. Bahrdt, Phys. Rev. Lett. 86, 3419 (2001).

${ }^{17}$ F. Nolting, A. Scholl, J. Stöhr, J.W. Seo, J. Fompeyrine, H. Siegwart, J.-P. Locquet, S. Anders, J. Lüning, E.E. Fullerton, M.F. Toney, M.R. Scheinfein, and H.A. Padmore, Nature (London) 405, 767 (2000).

${ }^{18}$ H. Ohldag, A. Scholl, F. Nolting, S. Anders, F.U. Hillebrecht, and J. Sthr, Phys. Rev. Lett. 86, 2878 (2001).

${ }^{19}$ H. Matsuyama, C. Haginoya, and K. Koike, Phys. Rev. Lett. 85, 646 (2000).

${ }^{20}$ W. Zhu, L. Seve, R. Sears, B. Sinkovic, and S.S.P. Parkin, Phys. Rev. Lett. 86, 5389 (2001).
${ }^{21}$ C. Kittel, in Introduction to Solid State Physics (Wiley, New York, 1971).

${ }^{22}$ M. G. Lagally, D. E. Savage, and M. C. Tringides, in Reflection High-Energy Electron Diffraction and Reflection Electron Imaging of Surfaces, edited by P.K. Larsen and P.J. Dobson (Plenum Press, New York, 1987), p. 139.

${ }^{23}$ J. Nogués, C. Leighton, and I.K. Schuller, Phys. Rev. B 61, 1315 (2000).

${ }^{24}$ Kentaro Takano, R.H. Kodama, A.E. Berkowitz, W. Cao, and G. Thomas, Phys. Rev. Lett. 79, 1130 (1997).

${ }^{25}$ R. Kannan and Mohindar S. Seehra, Phys. Rev. B 35, 6847 (1987).

${ }^{26}$ H. Kishi, Y. Kitade, Y. Miyake, A. Tanaka, and K. Kobayashi, IEEE Trans. Magn. 32, 3380 (1996).

${ }^{27}$ S. Soeya, H. Hoshiya, M. Fuyama, and S. Tadokoro, J. Appl. Phys. 80, 1006 (1996).

${ }^{28}$ S. Soeya, H. Hoshiya, R. Arai, and M. Fuyama, J. Appl. Phys. 81, 6488 (1997).

${ }^{29}$ A. Mougin, T. Mewes, M. Jung, D. Engel, A. Ehresmann, H. Schmoranzer, J. Fassbender, and B. Hillebrands, Phys. Rev. B 63, 060409 (2001).

${ }^{30} \mathrm{~J}$. Nogués (private communication).

${ }^{31}$ T.J. Moran, J.M. Gallego, and Ivan K. Schuller, J. Appl. Phys. 78, 1887 (1995).

${ }^{32}$ J. Nogués, T.J. Moran, D. Lederman, Ivan K. Schuller, and K.V. Rao, Phys. Rev. B 59, 6984 (1999).

${ }^{33}$ P.J. van der Zaag, Y. Ijiri, J.A. Borchers, L.F. Feiner, R.M. Wolf, J.M. Gaines, R.W. Erwin, and M.A. Verheijen, Phys. Rev. Lett. 84, 6102 (2000).

${ }^{34}$ D. Stauffer and A. Aharoni, in Percolation Theory (Taylor \& Francis, London, 1991).

${ }^{35}$ C. Schlenker, S.S.P. Parkin, J.C. Scott, and K. Howard, J. Magn. Magn. Mater. 54-57, 801 (1986).

${ }^{36}$ S.G.E. te Velthuis, A. Berger, G.P. Felcher, B.K. Hill, and E. Dan Dahlberg, J. Appl. Phys. 87, 5046 (2000).

${ }^{37}$ T. Ambrose and C.L. Chien, J. Appl. Phys. 83, 7222 (1998).

${ }^{38}$ J. Nogués, D. Lederman, T.J. Moran, and Ivan K. Schuller, Phys. Rev. Lett. 76, 4624 (1996)

${ }^{39}$ C. Leighton, J. Nogués, H. Suhl, and Ivan K. Schuller, Phys. Rev. B 60, 12837 (1999).

${ }^{40}$ P. Miltényi, M. Gierlings, M. Bamming, U. May, G. Güntherodt, J. Nogués, M. Gruyters, C. Leighton, and Ivan K. Schuller, 
Appl. Phys. Lett. 75, 2304 (1999).

${ }^{41}$ K.T.-Y. Kung, L.K. Louie, and G.L. Gorman, J. Appl. Phys. 69, 5634 (1991).

${ }^{42}$ O. Allegranza and M.-M. Chen, J. Appl. Phys. 73, 6218 (1993).

${ }^{43}$ U. Nowak, A. Misra, and K.D. Usadel, J. Appl. Phys. 89, 7269 (2001).

${ }^{44}$ J. Nogués, T.J. Moran, D. Lederman, Ivan K. Schuller, and K.V. Rao, Phys. Rev. B 59, 6984 (1999).

${ }^{45}$ J. Nogués, D. Lederman, T.J. Moran, Ivan K. Schuller, and K.V. Rao, Appl. Phys. Lett. 68, 3186 (1996).

${ }^{46}$ T.J. Moran, J.M. Gallego, and Ivan K. Schuller, J. Appl. Phys. 78, 1887 (1995).

${ }^{47}$ C.-M. Park, K.-I. Min, and K.H. Shin, IEEE Trans. Magn. 32, 3422 (1996).

${ }^{48}$ C.-M. Park, K.-I. Min, and K.H. Shin, J. Appl. Phys. 79, 6228 (1996).
${ }^{49}$ D.-H. Han, J.-G. Zhu, J.H. Judy, and J.M. Sivertsen, J. Appl. Phys. 81, 340 (1997).

${ }^{50}$ D.G. Hwang, S.S. Lee, and C.M. Park, Appl. Phys. Lett. 72, 2162 (1998).

${ }^{51}$ U. Nowak, A. Misra, and K.D. Usadel, J. Magn. Magn. Mater. 240, 243 (2002).

${ }^{52}$ S. Soeya, S. Nakamura, T. Imagawa, and S. Narishige, J. Appl. Phys. 77, 5838 (1995).

${ }^{53}$ R.D. McMichael, M.D. Stiles, P.J. Chen, and W.F. Egelhoff, Jr., Phys. Rev. B 58, 8605 (1998).

${ }^{54}$ M.R. Fitzsimmons, P. Yashar, C. Leighton, Ivan K. Schuller, J. Nogués, C.F. Majkrzak, and J.A. Dura, Phys. Rev. Lett. 84, 3986 (2000).

${ }^{55}$ C. Leighton, M.R. Fitzsimmons, P. Yashar, A. Hoffmann, J. Nogués, J. Dura, C.F. Majkrzak, and Ivan K. Schuller, Phys. Rev. Lett. 86, 4394 (2001). 